\title{
Graviton decay to 2 photons at the LHC
}

\author{
Simon Dalley ${ }^{1}$ \\ Department of Physics, Southern Methodist University \\ Dallas, TX 75275-0175, U.S.A. \\ E-mail: sdalley@physics.smu.edu
}

Standard Model production of $\gamma \gamma$ has been studied theoretically at hadron colliders by Balázs et al. [1] by developing the program RESBOS that computes the fully differential cross section typically in NLO QCD and at NLL resummation of initial state radiation. In collaboration with P. Nadolsky, our goal is to add to RESBOS the effect of an intermediate massive spin 2 particle $\mathrm{B}$, coupling to the energy momentum tensor, produced via the Drell-Yan process in $\mathrm{pp} \rightarrow \mathrm{B} \rightarrow \gamma \gamma \mathrm{X}$. This will help provide a model-independent spin determination of any new boson discovered at the LHC, since the $\gamma \gamma$ decay channel, though usually rare, is clean and shows pronounced angular variation. This note note presents the motivation and framework.

Light Cone 2010 - LC2010

Valencia, Spain

June 14-18, 2010

1 In collaboration with Pavel Nadolsky 


\section{Angular Variation}

The center-edge asymmetry $\sigma_{\mathrm{CE}}$ was used by Osland et al. [2] to quantify angular variation in the cross-section $\sigma\left(R_{l l}\right)$ for $\mathrm{pp} \rightarrow \mathrm{B} \rightarrow l l \mathrm{X}$ Drell-Yan dilepton production

$$
\sigma_{\mathrm{CE}} \equiv\left[\int_{-z^{*}}^{z^{*}}-\left(\int_{-z_{\text {cut }}}^{-z^{*}}+\int_{z^{*}}^{z_{\text {cut }}}\right)\right] \frac{\mathrm{d} \sigma\left(R_{l l}\right)}{\mathrm{d} z} \mathrm{~d} z .
$$

Here, $z=\cos \theta^{*}$, where $\theta^{*}$ is the polar angle of the dileptons in the rest frame of intermediate boson $\mathrm{B}$, and $z_{\text {cut }}$ is the limit of experimental acceptance. To get a rough idea, assuming no cuts or Standard Model background, and using only gluon fusion in LO QCD to produce B, we find the following comparison of angular variation for $l l$ and $\gamma \gamma$ final states:

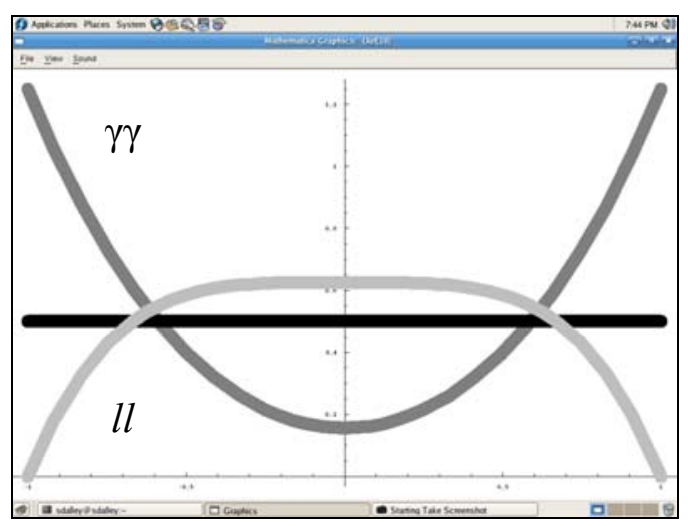

Fig.1(a) d $/ \mathrm{d} z$ v. $z$

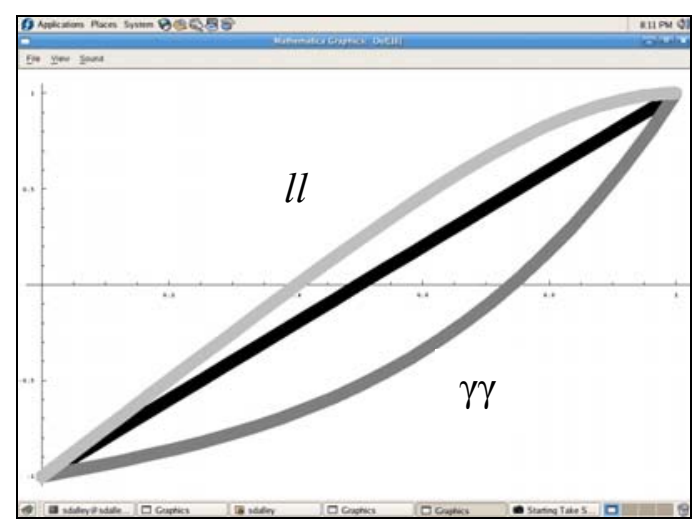

Fig.1(b) $\sigma_{\mathrm{CE}}$ V. $z^{*}$

The (light) gray curves are for spin-2 B decaying to $(l l) \gamma \gamma$. The isotropic decay of a scalar B is shown in black for reference. We see that the angular variation is more pronounced in $\gamma \gamma$, making it easier to distinguish tensor from scalar. Moreover, a vector B does not decay to $\gamma \gamma$, but does go to $l l$. For most collision energies and models, $\sigma\left(R_{l l}\right)$ is significantly larger than $\sigma$ $\left(R_{\gamma \gamma}\right)$, but the backgrounds are larger and identification more troublesome for the former.

\subsection{Helicity decomposition}

To include cuts, Standard Model backrounds, and the full PDFs, at NLO QCD and NLL resummation for $\sigma\left(R_{\gamma \gamma}\right)$, work is in progress to modify RESBOS. Consider the transition matrix element squared for di-photon production summed over final photon polarizations $\sigma=\{-1,0,1\}$, $\sum_{\sigma, \sigma^{\prime}}\left|H_{\mu v} \Delta^{\mu v \alpha \beta} P_{\alpha \beta}^{\sigma \sigma^{\prime}}\right|^{2} . H_{\mu v}$ and $P_{\alpha \beta}$ are hadron and photon tensors, $\Delta^{\mu v \alpha \beta}=\mathrm{i} B^{\mu \nu \alpha \beta}\left(q^{2}-M^{2}\right)^{-1}$ is the spin-2 B propagator for momentum $q$ and mass $M$. For proton momenta $p_{1}, p_{2}$, photon momenta $k_{1}, k_{2}$, with $k=k_{1}-k_{2}$,

$$
H_{\mu \nu} H_{\mu^{\prime} v^{\prime}}{ }^{*}=\int \mathrm{d}^{4} x \mathrm{e}^{\mathrm{i} q \cdot x}<p_{1} p_{2}\left|T_{\mu \nu}(x) T_{\mu^{\prime} v},(0)\right| p_{1} p_{2}>\quad, \quad B_{\mu v \alpha \beta}=1 / 2\left(g_{\mu \alpha} g_{v \beta}+g_{\mu \beta} g_{\alpha v}\right)+\ldots,
$$




$$
\begin{aligned}
4 \sum_{\sigma, \sigma^{\prime}} P_{\alpha \beta}^{\sigma \sigma^{\prime}{ }_{\alpha \beta} P_{\alpha^{\prime} \beta^{\prime}}{ }^{*}=} & q^{4}\left(g_{\alpha \alpha^{\prime}} g_{\beta \beta^{\prime}}-1 / 2 g_{\alpha \beta} g_{\alpha^{\prime} \beta^{\prime}}\right)+2 q^{2} g_{\alpha \alpha^{\prime}}\left(k_{\beta} k_{\beta^{\prime}}-q_{\beta} q_{\beta^{\prime}}\right) \\
& -1 / 2 q^{2}\left(g_{\alpha \beta} k_{\alpha^{\prime}} k_{\beta^{\prime}}+g_{\alpha^{\prime} \beta^{\prime}} k_{\alpha} k_{\beta}\right)+1 / 2\left(k_{\beta} k_{\alpha} q_{\alpha^{\prime}}+k_{\beta^{\prime}} k_{\alpha^{\prime}} q_{\alpha} q_{\beta}\right) \\
& -2 k_{\beta} k_{\beta^{\prime}} q_{\alpha^{\prime}} q_{\alpha}+1 / 2\left(k_{\alpha} k_{\alpha^{\prime}} k_{\beta} k_{\beta^{\prime}}+q_{\alpha^{\prime}} q_{\alpha} q_{\beta} q_{\beta^{\prime}}\right)+\mathrm{S}
\end{aligned}
$$

We neglected terms in $B$ that do not contribute to $|H B P|^{2}$ for on-shell photons. S adds the $\alpha \leftrightarrow$ $\beta$ and/or $\alpha^{\prime} \leftrightarrow \beta^{\prime}$ expressions. The framework of RESBOS uses a helicity decomposition and factorization possible in the case of an irreducible intermediate state. We have the completeness condition $B_{\mu v \alpha \beta}=\Sigma_{s} \varepsilon_{\mu \nu}^{s} \varepsilon_{\alpha \beta}^{s}{ }^{*}$, where $\varepsilon_{\mu v}^{s}$ are the traceless, transverse, orthogonal spin 2 polarization tensors, and $s$ are the polarizations $\{-2,-1,0,1,2\}$ of spin 2 . We can then write

$$
\left(q^{2}-M^{2}\right)^{2} \sum_{\sigma, \sigma^{\prime}}\left|H_{\mu \nu} \Delta^{\mu \nu \alpha \beta} P^{\sigma \sigma^{\prime}}{ }_{\alpha \beta}\right|^{2}=\sum_{s, s^{\prime}} H_{\left(s, s^{\prime}\right)} P_{\left(s, s^{\prime}\right)}
$$

where

$$
H_{\left(\mathrm{s}, s^{\prime}\right)}=H^{\mu v} H^{\mu^{\prime} v^{\prime *}} \varepsilon_{\mu \nu}^{s} \varepsilon_{\mu^{\prime} v^{v^{\prime}}}^{s^{\prime}}, \quad, \quad P_{\left(s, s^{\prime}\right)}=\sum_{\sigma, \sigma^{\prime}} P^{\sigma \sigma^{\prime} \alpha \beta} P^{\sigma \sigma^{\prime} \alpha^{\prime} \beta^{\prime *}} \varepsilon_{\alpha \beta}^{s^{*}}{ }^{*} \varepsilon^{s^{\prime} \alpha^{\prime} \beta^{\prime}} .
$$

$H_{\left(\mathrm{s}, \mathrm{s}^{\prime}\right)}$ are computed by subroutines in RESBOS at the parton level to include resummation effects, NLO QCD corrections and PDFs. They depend only upon $q$, transverse momentum $q_{\mathrm{T}}$ and rapidity $y$ of B. Symmetries under $s \leftrightarrow-s$ and $s \leftrightarrow s$ 'imply that nine of the twenty-five $P_{\left(s, s^{\prime}\right)}$ are independent. They may be parameterized by the following (non-unique) angular functions

$$
\begin{aligned}
& P_{(-2,2)}=1 / 4 q^{4}\left[\sin ^{4} \theta^{*}\right] \operatorname{exp~i4} \varphi^{*} \\
& P_{(-1,2)}=1 / 2 q^{4}\left[\sin ^{3} \theta^{*} \cos \theta^{*}\right] \operatorname{exp~i3\varphi ^{*}} \\
& P_{(-1,1)}=q^{4}\left[\sin ^{4} \theta^{*}\right] \operatorname{exp~i} 2 \varphi^{*} \\
& P_{(0,2)}=-\left(q^{4} / 2 \sqrt{6}\right)\left(\left[\sin ^{2} \theta^{*}\right]+\left[\sin ^{2} \theta^{*} \cos ^{2} \theta^{*}\right]\right) \exp i 2 \varphi^{*} \\
& P_{(1,2)}=1 / 2 q^{4}\left(4\left[\sin \theta^{*} \cos \theta^{*}\right]-\left[\sin ^{3} \theta^{*} \cos \theta^{*}\right]\right) \operatorname{exp~i\varphi ^{*}} \\
& P_{(0,1)}=\left(2 q^{4} / \sqrt{ } 6\right)\left(3\left[\sin \theta^{*} \cos \theta^{*}\right]-\left[\sin ^{3} \theta^{*} \cos \theta^{*}\right] \exp \mathrm{i} \varphi^{*}\right. \\
& P_{(2,2)}=1 / 4 q^{4}\left(8\left[\cos ^{2} \theta^{*}\right]+\left[\sin ^{4} \theta^{*}\right]\right) \\
& P_{(1,1)}=q^{4}\left(\left[\sin ^{2} \theta^{*}\right]+\left[\sin ^{2} \theta^{*} \cos ^{2} \theta^{*}\right]\right) \\
& P_{(0,0)}=\left(q^{4} / 6\right)\left(16-7\left[\sin ^{2} \theta^{*}\right]-\left[\sin ^{2} \theta^{*} \cos ^{2} \theta^{*}\right]\right)
\end{aligned}
$$

The imaginary parts cancel in $|H B P|^{2}$ once the sum over $\left(s, s^{\prime}\right)$ is done.

\section{References}

[1] C. Balázs, E.L. Berger, P.M. Nadolsky, and C-P. Yuan, Calculation of prompt diphoton production cross sections at Tevatron and LHC energies, Phys. Rev. D76 013009 (2007) [hep$\mathrm{ph} / 0704.0001]$

[2] P. Osland, A.A.Pankov, N. Paver, and A.V. Tsytrinov, Spin identification of the Randall-Sundrum resonance in lepton-pair production at the LHC, Phys. Rev. D78 035008 (2008) [hep$\mathrm{ph} / 0805.2734]$ 